\title{
Prediction of the Costs of the Missile Equipment Based on SVR
}

\author{
Zheng Xianzhu $^{1, \mathrm{a}}$, Du Jiaxing ${ }^{1, \mathrm{~b}}$, Yuan Jianguang ${ }^{2, \mathrm{c}}$, Tong Jifeng ${ }^{1, \mathrm{~d}}$, \\ Tian Jiaping, ${ }^{1, \mathrm{e}}$ \\ ${ }^{1}$ Department of Scientific Research, Academy of Armored Forces \\ Engineering, Beijing,100072, China \\ ${ }^{2}$ Institute of Nonlinear Science, Academy of Armored Force Engineering, \\ Beijing 100072, China \\ ${ }^{a}$ zhuzi_zorro@sina.com, ${ }^{b}$ \\ dht12004@163.com, ${ }^{c}$ dych1013@sina.com, ${ }^{d}$ tongjifeng@163.com, ${ }^{e} 57340$ \\ 507@qq.com
}

\begin{abstract}
The difficulty of the prediction of missile equipment costs lies in the small sample data, and the sample data have the complicated non-linear characteristics. By analyzing the influence of parameters of missile equipment costs, SVR is proposed to predict the missile equipment costs model, and Taking a certain missile equipment cost as an example to verify the equipment costs. The calculation results show that this method has better prediction precision and higher prediction accuracy.
\end{abstract}

Keywords: Missile equipment, SVR, Cost forecast.

\section{Introduction}

The traditional methods of the prediction of missile equipment costs in practice are mainly the engineering estimation method and parameter estimation method, analogy method and expert estimation method[1]. Among them, the engineering estimation method can get more detailed and accurate cost estimates, but it is also very troublesome job. For example, one of the important parts in estimation of missile equipment costs, often need complex calculation. Parameter estimation method is to select appropriate parameters to construct 
explicit functional form, usually it implies a linear relationship hypothesis, but the real problem lies in many nonlinear relationships. The defect of analogy method and expert estimation method lies in the estimate precision is not high. At the same time, as a highly nonlinear problem description ability of the modeling tool, neural network prediction field has been applied widely in missile equipment costs[2]. But the neural network technology also has some inherent defects: it do not have unified criterion to determine the network structure; it have certain requirements on the sample in the quantity and quality; it is Prone to have over fitting and poor model generalization ability, and so on. Because the sample is limited, missile equipment cost prediction problem is a typical small sample learning problem; many methods are difficult to achieve the ideal effect[3-4]. Therefore, in order to provide accurate information promptly to decision makers, we must find a prediction method for economic, quick and accurate scientific prediction of missile equipment costs.

Statistical learning theory (SLT) is a machine learning rule of a specialized research in small samples under the theory established by Vapnik. Support vector machine (SVM) is developed on the basis of this theory into a new classification and regression tools. Support vector regression is mainly including E-SVR presented by Vapnik and $v$-SVR proposed by Schlkopf [5-6]. $\varepsilon$-SVR control algorithm is hoping to achieve precision by predetermined $\varepsilon$, and $v$-SVR to minimizes, so as to ensure that the algorithm can achieve the highest precision. Support vector machine to improve the generalization ability through the structural risk minimization principle, solves the small sample, non-linear, high dimension, local minimum and so on, it has been widely used [7-10]. Therefore, this paper attempts to use support vector regression theory to establish the prediction model of missile equipment cost, to have the cost forecasting, and to achieve the good effect.

\section{The basic principle of $v$-SVR support vector regression}

$$
\text { A training set } T=\left\{\left(x_{1}, y_{2}\right), \mathrm{L}\left(x_{l}, y_{l}\right)\right\} \in(X \times Y)^{l} \quad \text {, among }
$$


them $\quad x_{i} \in X=R^{n}, y_{i} \in Y=R, i=1,2, \mathrm{~L}, l \quad$, which uses the nonlinear mapping of $\phi(x)$ the input vector ${ }^{X}$ is mapped to the feature space, and then linear regression in high dimensional feature space, construct the regression function

$$
f(x)=\omega^{T} \cdot \phi(x)+b
$$

Among them $\omega$ and $\boldsymbol{b}$ respectively denote the weight vector and bias. The introduction of slack variables $\xi^{(*)}=\left(\xi_{1}, \xi_{1}^{*}, \mathrm{~L}, \xi_{l}, \xi_{l}^{*}\right)^{T}$ and the penalty parameter $C$, and to construct the two times planning original problem:

$$
\min _{\omega \in R^{n}, \xi^{(*)} \in R^{2 l}, b \in R} \frac{1}{2}\|\omega\|^{2}+C \cdot\left(v \varepsilon+\frac{1}{l} \sum_{i=1}^{l}\left(\xi_{i}+\xi_{i}^{*}\right)\right)
$$

s.t. $\left(\omega^{T} x_{i}+b\right)-y_{i} \leq \varepsilon+\xi_{i}, \quad i=1,2, \mathrm{~L}, l$

$$
\begin{aligned}
y_{i}-\left(\omega^{T} x_{i}+b\right) \leq \varepsilon+\xi_{i}^{*}, \quad i=1,2, \mathrm{~L}, l \\
\varepsilon, \xi_{i}, \xi_{i}^{*} \geq 0, \quad i=1,2, \mathrm{~L}, l
\end{aligned}
$$

Type (2) for the dual problem:

$$
\begin{aligned}
& \min _{\alpha^{(*)} \in R^{2 l}} \frac{1}{2} \sum_{i, j=1}^{l}\left(\alpha_{i}^{*}-\alpha_{i}\right)\left(\alpha_{j}^{*}-\alpha_{j}\right) K\left(x_{i}, x_{j}\right)-y_{i} \sum_{i=1}^{l}\left(\alpha_{i}^{*}-\alpha_{i}\right) \\
& \text { s.t. } \quad \sum_{i=1}^{l}\left(\alpha_{i}^{*}-\alpha_{i}\right)=0 \\
& 0 \leq \alpha_{i}^{*}, \alpha_{i} \leq \frac{C}{l}, i=1,2, \mathrm{~L}, l \\
& \sum_{i=1}^{l}\left(\alpha_{i}^{*}-\alpha_{i}\right) \leq C \cdot v
\end{aligned}
$$

$\alpha, \alpha^{*}$ is the Lagrange multiplier, $K\left(x_{i}, x_{j}\right)$ as a satisfying Mercer condition symmetric kernel function, kernel function are commonly used polynomial kernel function (Polynomial), radial basis function (RBF), Sigmoid kernel function etc. 
v-SVR algorithm steps:

1) A training set $T=\left\{\left(x_{1}, y_{2}\right), \mathrm{L}\left(x_{l}, y_{l}\right)\right\} \in(X \times Y)^{l}$, the

$x_{i} \in X=R^{n}, y_{i} \in Y=R, i=1,2, \mathrm{~L}, l$.

2) Select the appropriate positive $v, C$, and kernel function $K\left(x, x^{\prime}\right)$.

3)Constructing and solving the optimization problem (3), to obtain the optimal solution $\bar{\alpha}=\left(\bar{\alpha}_{1}, \bar{\alpha}_{1}^{*}, \mathrm{~L}, \bar{\alpha}_{l}, \bar{\alpha}_{l}^{*}\right)^{T}$.

4) To construct the decision-making function

$$
f(x)=\sum_{i=1}^{l}\left(\bar{\alpha}_{i}^{*}-\bar{\alpha}_{i}\right) K\left(x_{i}, x\right)+\bar{b}
$$

The choice is located in the open interval in $\bar{\alpha}_{j}$ or $\bar{\alpha}_{k}^{*}$. Order

$$
\bar{b}=\frac{1}{2}\left[y_{i}+y_{k}-\left(\sum_{i=1}^{l}\left(\bar{\alpha}_{i}^{*}-\overline{\alpha_{i}}\right) K\left(x_{i}, x_{j}\right)+\sum_{i=1}^{l}\left(\bar{\alpha}_{i}^{*}-\overline{\alpha_{i}}\right) K\left(x_{i}, x_{k}\right)\right.\right.
$$

\section{Prediction of missile equipment costs}

Missile initial quality, warhead quality, maximum speed, maximum range and the missile cost are the key factor that they all have affects on the missile equipment costs. The literature [12] selects 10 groups of missile equipment cost model data to have the cost analysis, the training sample and test sample data as shown in Table 1. The first 9 groups set as training samples for existing equipment, the tenth group as the simulation samples for research in equipment, and the cost forecast by using SVR model, and it compared with the actual value. 
Table 1 The cost of the sample data of ground to air missile

\begin{tabular}{ccccccc}
\hline No. & Initial quality & $\begin{array}{c}\text { Warhead qu } \\
\text { ality }\end{array}$ & $\begin{array}{c}\text { The } \\
\text { maximum } \\
\text { quality }\end{array}$ & $\begin{array}{c}\text { The } \\
\text { maximum } \\
\text { range / sea } \\
\text { mile }\end{array}$ & $\begin{array}{c}\text { Missile cost/ } \\
\text { billion } \\
\text { dollars }\end{array}$ & $\begin{array}{c}\text { Cumulatie } \\
\text { cost / billion } \\
\text { dollars }\end{array}$ \\
\hline 1 & 1000 & 100 & 6 & 70 & 3 & 19 \\
2 & 1358.2 & 61.2 & 3 & 127.9 & 2.8 & 18 \\
3 & 630 & 50 & 2.5 & 25 & 1.2 & 7.2 \\
4 & 63.5 & 5.9 & 1.6 & 9.3 & 0.55 & 3.2 \\
5 & 87 & 14 & 2.3 & 10 & 1 & 5 \\
6 & 86.2 & 4.5 & 2.5 & 5 & 0.9 & 3.7 \\
7 & 447 & 60 & 5 & 200 & 4 & 30.5 \\
8 & 78 & 4 & 2.3 & 14.5 & 0.95 & 4.5 \\
9 & 204 & 27 & 3.5 & 44.5 & 1.5 & 10 \\
10 & 625 & 50 & 2.5 & 40 & 1.3 & 7.9 \\
\hline
\end{tabular}

We set the missile initial quality, quality, warhead maximum speed, maximum range, the missile cost as the input parameters, and the cumulative cost as output, the nuclear function takes the radial basis kernel function (RBF) $\quad K\left(x, x^{\prime}\right)=\exp \left(-\gamma\left\|x-x^{\prime}\right\|^{2}\right) \quad$, among them $\gamma=0.5$, the penalty coefficient $C=1, v=0.5, \varepsilon=0.001$, we established the model of $v$-SVR, and the measured value and fitted value comparison of missile cost as shown in Table 2 . As can be seen, SVR model to fit the average value of relative error is only $2.59 \%$, the coefficient of determination of 0.99 , the fitting effect is good. 
Table 2 Surface to air missile cost measured value and quasi comparison table of values

\begin{tabular}{cccc}
\hline No. & $\begin{array}{c}\text { The measured } \\
\text { value }\end{array}$ & The fitted values & The relative error \\
\hline 1 & 19 & 19.0086 & 0.0453 \\
2 & 18 & 17.9962 & -0.0211 \\
3 & 7.2 & 7.1983 & -0.0236 \\
4 & 3.2 & 3.2164 & 0.5125 \\
5 & 5 & 4.909 & -1.82 \\
6 & 3.7 & 4.4554 & 20.4162 \\
7 & 30.5 & 30.4898 & -0.0334 \\
8 & 4.5 & 4.4829 & -0.38 \\
9 & 10 & 10.0086 & 0.086
\end{tabular}

Cost forecast of 10 missile armament carried by this model; get the forecasting results as shown in Table 3

Table 3 Predicted surface to air missile cost

\begin{tabular}{cccc}
\hline No. & $\begin{array}{c}\text { The measured } \\
\text { value }\end{array}$ & The fitted values & The relative error \\
\hline 10 & 7.9 & 7.8121 & -1.1126
\end{tabular}

The practical results show that SVR has good generalization ability in the case of limited samples, and has a certain universality, it can be used as a suitable method and it should be popularized.

\section{Conclusions}

Support vector regression is a statistical learning model by the principle of structural risk minimization instead of empirical risk minimization principle, has perfect theory basis. Based on the analysis of missile equipment cost data, we use support vector regression model to build missile equipment cost theory and 
method. From the example above, it can be seen that the support vector machine methods have better calculation accuracy and have stronger generalization ability in dealing with nonlinear problems.

\section{References}

[1] J.Y. Zhu, X.B. Zhang, H.X. Zhang, J. Pei. Systems Engineering and Electronics, Vol.25(2003), p. 701-703 (In Chinese)

[2]]Y. Y. Jiang, K. Meng, H. X. Zhang, X. Xu. Computer Engineering, Vol.5(2007), p. 154-155 (In Chinese)

[3] M. He. J. Air Force Engineering University, Vol.1(2008), p. 22-25 (In Chinese)

[4] F. Guo, H. X. Zhang, S. A. Li, Q. Zhang. J. Air Force Engineering University, Vol.6(2005), p. 10-15 (In Chinese)

[5] Vapnik V N. On Neural Network, Vol.5(1999), p.988-999.

[6] Vapnik V N. The nature of statistical learning theory, Springer Verlag, New York(1995).

[7] Schölkopf B et al. Neural Computation, Vol.12(2000), p.1207-1245.

[8] Q. Chen, M.C. Wu, Y.J. Xue et al. Computer Engineering and Applications, Vol.21(2009), p.235-238(In Chinese)

[9] D.Q. Li. Ship \& Ocean Engineering, Vol.2(2009), p.104-106(In Chinese)

[10] C.B. Lian, Y.J. Lian et al. J. XI'an University of Science and Technology, Vol.4(2008), p.707-710(In Chinese) 